\title{
Neuroradiologie: Für die Zukunft beste Aussichten
}

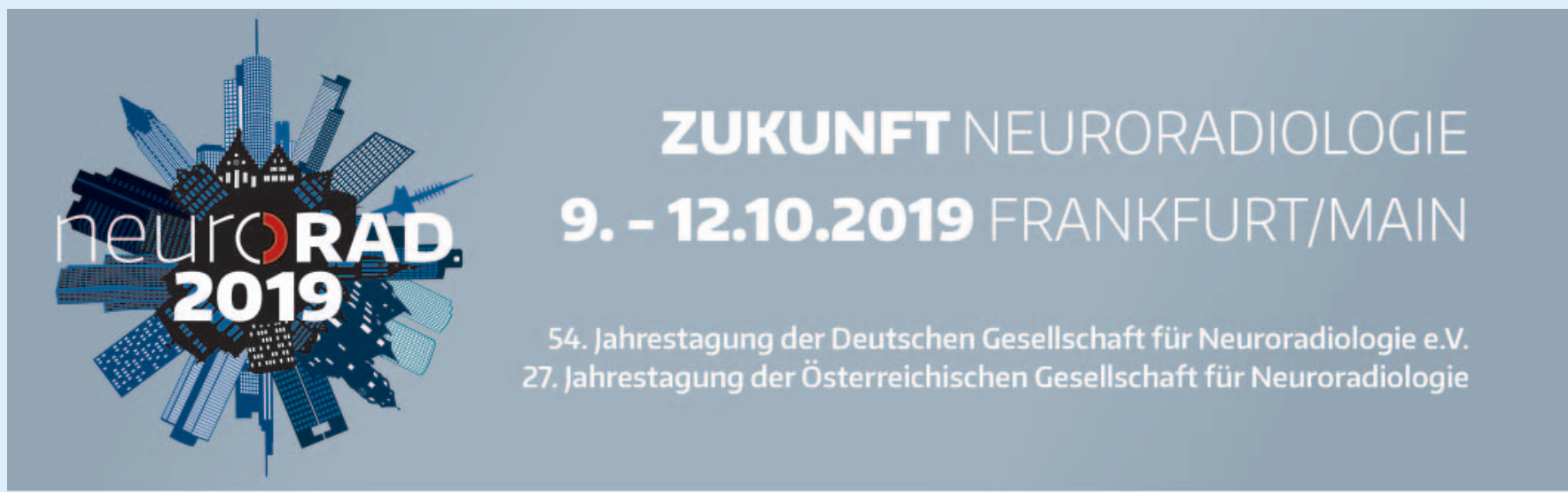

Die 54. Jahrestagung der Deutschen Gesellschaft für Neuroradiologie und gleichzeitig die 27. Jahrestagung der Österreichischen Gesellschaft für Neuroradiologie findet in 2019 vom 9. bis 12. Oktober im Kap Europa in Frankfurt am Main statt. Längst der Höhepunkt des neuroradiologischen Kongressjahres, wartet der diesjährige „neuroRAD“ mit Altbewährtem, aber auch zahlreichen Neuerungen auf. Die Kongresspräsidenten Prof. Dr. Claus Zimmer aus München und Prim. Dr. Johannes Trenkler aus Linz berichten im Interview, welche thematischen Schwerpunkte sie gelegt haben, worauf sich die Teilnehmenden beim neuen Format der Power Pitches freuen können und was hinter dem Kongressmotto steckt.

\section{„Zukunft Neuroradiologie“ lautet das Motto des neuroRAD 2019. Warum haben Sie gerade dieses gewählt?}

Prof. Dr. Claus Zimmer: Die Medizin ganz allgemein und natürlich auch die Neuroradiologie befinden sich derzeit in einer Umbruchphase. Künstliche Intelligenz, Maschinelles Lernen und digitale Medizin sind dafür nur einige wenige Stichworte. Dem wollen wir mit unserem Kongress Rechnung tragen. Wir werden zeigen, dass die Neuroradiologie als Querschnittsfach in Diagnostik und Therapie von ZNS-Erkrankungen eine große Zukunft hat und wir wollen uns schwerpunktmäßig damit beschäftigen, wie denn die Zukunft aussehen könnte. Die Neuroradiologie war bei der Einführung von neuen Technologien schon immer ganz vorne mit dabei.

Prim Dr. Johannes Trenkler: „Zukunft Neuroradiologie“ hat für uns zwei Bedeutungen:

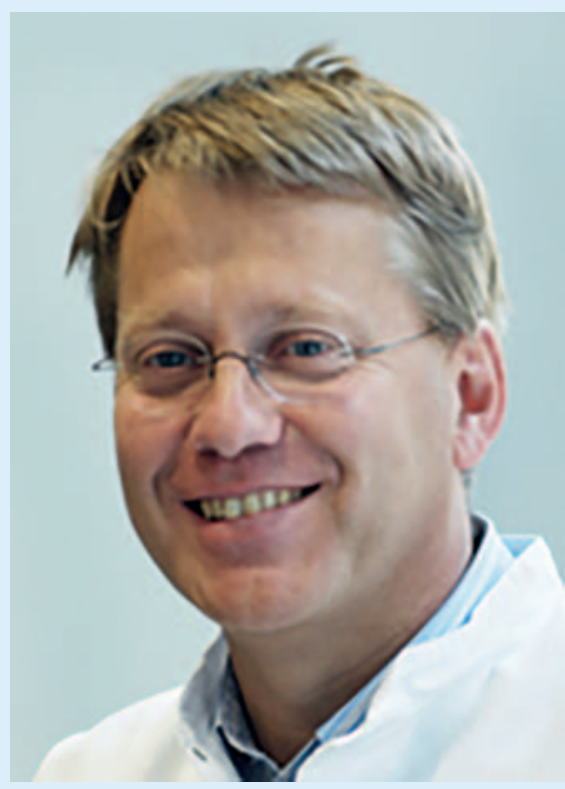

Prof. Dr. Claus Zimmer, Technische Universität München

Zum einen sollen mögliche Veränderungen durch zunehmenden Einfluss neuer Entwicklungen wie künstlicher Intelligenz mit allen Risiken und Chancen thematisiert werden. Wir Neuroradiologen haben über viele Jahre eine sehr große Anzahl an elektronischen Bild- und Befunddaten in unseren Archivsystemen abgelegt, die mit die wertvollste Grundlage zur Entwicklung von $\mathrm{KI}$-Anwendungen in der Medizin darstellen. Durch den routinemäßigen Einsatz von Auswertesoftware für diese Bilddaten ist die Neuroradiologie schon längst in diese Entwicklung involviert und hat so für die Zukunft beste Voraussetzungen. Zum anderen bedeutet es, dass die Neuroradiologie als Querschnittsfach für die Medizin der Zukunft eine zunehmend wichtige Rolle

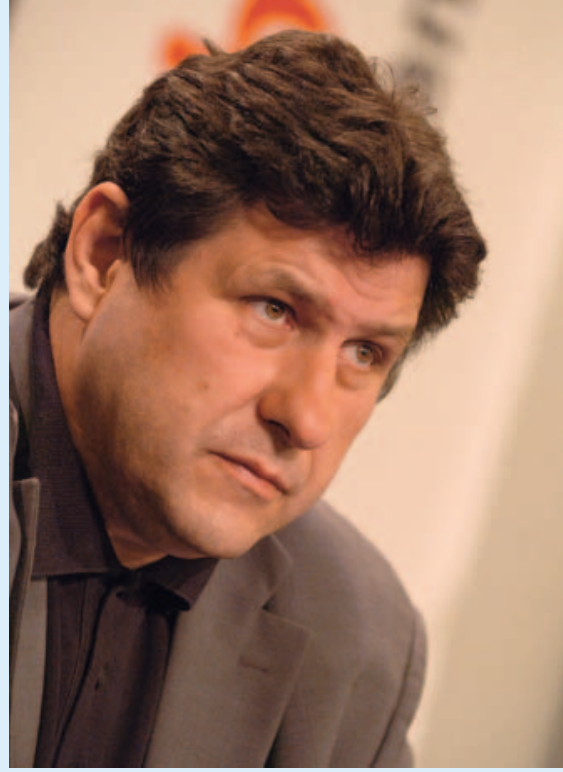

Prim Dr. Johannes Trenkler, Kepler Universitätsklinikum Linz

spielt, unter anderem durch die rasante Entwicklung der minimal-invasiven Schlaganfallbehandlung. Wir wollen vermitteln, dass der Neuroradiologie als integraler Bestandteil der neuromedizinischen Fächer eine zunehmend wichtige Rolle zukommt.

Was sind die Schwerpunktthemen dieses Jahres und wer wird für die Keynote-Lectures eingeladen?

Zimmer: Als Schwerpunkte des diesjährigen Kongresses haben wir als übergeordnetes Thema „Künstliche Intelligenz“ gewählt und ferner die großen krankheitsbezogenen Themen Neuroonkologie, entzündliche ZNS-Erkrankungen, Neurodegeneration, Wirbelsäule und natürlich die zerebrovaskulären 
Erkrankungen. Dementsprechend haben wir für diese Erkrankungen ausgewiesene Referenten eingeladen, die jeweils Keynote-Vorträge halten werden. So freuen wir uns ganz besonders, dass Herr Prof. Dr. Otmar Wiestler, einer der „Urväter“ der WHO-Klassifikation der Hirntumoren und jetziger Präsident der Helmholtz-Gemeinschaft deutscher Forschungszentren, zugesagt hat, ebenso wie Dr. Mayank Goyal aus Calgary, der über die neuesten Entwicklungen zur Thrombektomie sprechen wird. Über die Keynote-Vorträge suchen wir auch den engen Dialog mit den Kollegen aus den benachbarten Fächern.

Erstmals bieten Sie in diesem Jahr die StAR-Lounge (Start-Up and Applied Research) an. Wen möchten Sie mit diesem Forum ansprechen und wie läuft der Wissenstransfer?

Trenkler: Mit diesem Format wollen wir innovative Start-Ups und Forschungsgruppen aus den Bereichen künstlicher Intelligenz, Datenmanagement, Robotik und virtueller Realität ansprechen und wir möchten, dass diese Initiativen ihre Anwendungen zu Diagnostik, minimal-invasiven Therapien, Befundung, Optimierung von Arbeitsabläufen und Ausbildung uns vorstellen. Besonders wichtig dabei ist uns der Austausch zwischen den klinisch tätigen Neuroradiologen und den Entwicklern bzw. Unternehmern, um die klinische Anwendung innovativer Entwicklungen zu fördern. Die Unternehmen können sich in Pitch-Sessions präsentieren, einem in der Branche gängigen, sehr dynamischen Vortrags-Format, das nicht nur kurz, sondern auch kurzweilig ist.

Zimmer: Ein ganzes Stockwerk des Kap Europa werden wir zur StAR-Lounge umfunktionieren. Neben den schon angesprochenen Pitch-Sessions werden dort aktiv Diskussionsrunden initiiert, beispielsweise zur finanziellen Vergütung von neuen Bild-basierten Auswertetools oder zu rechtlichen Aspekten bei der Einführung von $\mathrm{KI}$ in die Krankenversorgung. Diese Diskussionsforen werden von entsprechenden Fachexperten moderiert. In Ergänzung dazu werden wir am Mittwoch zum Kongressauftakt erstmals einen ganztägigen, anwendungsorientierten Workshop zum Thema „Künstliche Intelligenz für Neuroradiologen“ anbieten. Dieser Workshop wird absolut „basic“ sein, keine speziellen Vorkenntnisse sind dafür erforderlich.
Auch für die jungen Kolleginnen und Kollegen wird es ein neues Präsentationsformat geben - die Power Pitches. Was darf man sich darunter vorstellen und warum haben Sie dieses Präsentationsformat ausgewählt?

Zimmer: Ein Manko der vergangenen Kongresse war aus meiner Sicht, dass junge motivierte Kollegen ihre größtenteils äußerst erfrischenden Vorträge häufig vor zum Teil fast leeren Hörsälen halten mussten. Dies wollen wir ändern. Es ist geplant, dass zu jedem Schwerpunktthema eine gewisse Anzahl von Vorträgen vor großem Publikum auch von unserem wissenschaftlichen Nachwuchs im Hauptvortragssaal gehalten wird. Dies geschieht als Power Pitch, in dem das Projekt innerhalb von kurzer Zeit zunächst einem großen Publikum schmackhaft gemacht werden soll, bevor es anschließend am Poster vertieft wird. Ich persönlich freue mich schon jetzt sehr auf diese Beiträge.

Was erwartet die Kongressteilnehmer in den Fit-für-den-Facharzt-Kursen?

Trenkler: Die Teilnehmer an den FFF-Kursen erwarten ausgesuchte und spannende Themen 
aus den Bereichen zerebrovaskuläre Erkrankungen und Interventionen, spinale Erkrankungen, Neuropädiatrie und Entzündungen. Beiträge über die Bildgebung bei Kopfschmerzen, Notfallbildgebung, ZNS-Veränderungen beim Glaukom sowie über Irrtümer und Fehler in der Neuroradiologie sollen den zukünftigen Fachärzten das Rüstzeug für die ersten Nachtdienste mitgeben. Hot Topics wie die aktuelle Diskussion zu MR-Kontrastmitteln sowie Hirntoddiagnostik werden ebenso thematisiert wie eine Einführung in den Befund der Zukunft. Für alle diese Themen und Beiträge konnten namhafte und kompetente Vortragende gewonnen werden.

\section{Auf welche Themen können sich MTRA freuen?}

Zimmer: Auch dieses Jahr wird es ein zweieinhalbtägiges Programm für MTRA und
MTRA-Schüler geben. Dieser Programmteil wird in Verantwortung der Vereinigung Medizinisch-Technischer Berufe in der Deutschen Röntgengesellschaft organisiert. Vorträge zum „Berufsbild der MTRA“, über „akute Notfälle im Nachtdienst und typische Krankheitsbilder, die die MTRA erkennen sollte" bis hin zum Thema Hygiene in der Neuroradiologie.

Trenkler: Am Samstag findet für MTRA, Studierende und junge Ärzte zudem die Stroke Rallye statt. Hier kann das für die Thrombektomie notwendige Material hands-on kennengelernt werden. An Flussmodellen lösen die Teilnehmenden dann konkrete Aufgaben.

\section{Vielen Dank für das Gespräch!}

Zweite German Stroke School auch für Mitglieder von DGNR und DeGIR!

Die Veranstaltung wird voraussichtlich mit 18 Unterrichtseinheiten für Modul E DeGIR zertifiziert.

Bereits zum zweiten Mal nach 2018 wird die German Stroke School auf dem neuroRAD angeboten. Zwei Tage intensives Training an Simulatoren und Flussmodellen, verbunden mit einem Lecture-Programm, das von ausgewiesenen Spezialisten abgehalten wird: Die German Stroke School wird auch in diesem Jahr eine herausragende Gelegenheit sein, die Thrombektomie beim akuten ischämischen Schlaganfall zu erlernen.

Alle Infos finden Sie hier: neurorad.de > 2nd German Stroke School 